\title{
GLUT12 deficiency during early development results in heart failure and a diabetic phenotype in zebrafish
}

\author{
Vanesa Jiménez-Amilburu ${ }^{\dagger}$, Susanne Jong-Raadsen', Jeroen Bakkers², \\ Herman $P$ Spaink and Rubén Marín-Juez ${ }^{1}$ \\ Institute of Biology, Leiden University, PO Box 9502, 2300 RA Leiden, The Netherlands \\ 'ZF-screens BV, J.H. Oortweg 19, 2333 CH Leiden, The Netherlands \\ ${ }^{2}$ Hubrecht Institute-KNAW and University Medical Center Utrecht and Interuniversity, 3584 CT Utrecht, \\ The Netherlands \\ ${ }^{\dagger} \mathrm{V}$ Jiménez-Amilburu is now at Department of Developmental Genetics, Max Planck Institute for Heart and \\ Lung Research, Ludwigstrasse 43, 61231 Bad Nauheim, Germany
}

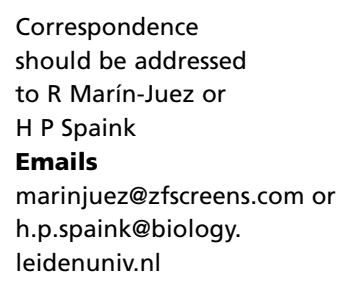

\begin{abstract}
Cardiomyopathies-associated metabolic pathologies (e.g., type 2 diabetes and insulin resistance) are a leading cause of mortality. It is known that the association between these pathologies works in both directions, for which heart failure can lead to metabolic derangements such as insulin resistance. This intricate crosstalk exemplifies the importance of a fine coordination between one of the most energy-demanding organs and an equilibrated carbohydrate metabolism. In this light, to assist in the understanding of the role of insulinregulated glucose transporters (GLUTs) and the development of cardiomyopathies, we have developed a model for glut12 deficiency in zebrafish. GLUT12 is a novel insulin-regulated GLUT expressed in the main insulin-sensitive tissues, such as cardiac muscle, skeletal muscle, and adipose tissue. In this study, we show that glut12 knockdown impacts the development of the embryonic heart resulting in abnormal valve formation. Moreover, glut12-deficient embryos also exhibited poor glycemic control. Glucose measurements showed that these larvae were hyperglycemic and resistant to insulin administration. Transcriptome analysis demonstrated that a number of genes known to be important in cardiac development and function as well as metabolic mediators were dysregulated in these larvae. These results indicate that glut12 is an essential GLUT in the heart where the reduction in glucose uptake due to glut12 deficiency leads to heart failure presumably due to the lack of glucose as energy substrate. In addition, the diabetic phenotype displayed by these larvae after glut12 abrogation highlights the importance of this GLUT during early developmental stages.
\end{abstract}

\author{
Key Words \\ - glut12 \\ - heart failure \\ - diabetes \\ - valve dysgenesis \\ - zebrafish
}

Journal of Endocrinology (2015) 224, 1-15

\section{Introduction}

Cardiovascular pathologies are a leading cause of mortality (Pagidipati \& Gaziano 2013). Heart failure constitutes the primary cause of death in developed countries and its occurrence increases rapidly in developing countries (Sliwa et al. 2005, Lopez et al. 2006). As the heart is one of the most energy demanding tissues, heart 
failure is tightly correlated with alterations in cardiac metabolism. In this regard, pathologies impacting metabolism and leading to alterations in glycemic control present one of the main risk factors for developing heart failure (Haslam \& James 2005). Specially, type 2 diabetes (T2D) is strongly associated with heart failure (Bell 2003a). Patients with T2D present an increased incidence of heart failure ranging from two- to fivefold higher than nondiabetic patients (Kannel \& McGee 1979). The main insulin-regulated glucose transporter (GLUT) expressed in peripheral tissues such as cardiac and skeletal muscle and adipose tissue is GLUT4 (Scheepers et al. 2004). In the presence of insulin, GLUT4 translocates to the plasma membrane, mediating the postprandial glucose uptake. Surprisingly, Glut4-deficient mice did not develop hyperglycemia, and skeletal muscle tissue showed an increased glucose uptake in response to insulin (Katz et al. 1995, Stenbit et al. 1996), suggesting that there might be other insulin-sensitive GLUTs still to be identified. Interestingly, years later when it was discovered, it was found that a newly discovered GLUT named GLUT12 is expressed in the main insulin-sensitive tissues and shares motifs that are known to be important for the regulation of GLUT4 in response to insulin (Rogers et al. 2002). Moreover, Stuart et al. (2009) demonstrated that in human myoblasts insulin stimulates GLUT12 translocation to the plasma membrane via PI3-K, in the same manner as GLUT4. In this light, recent data have shown that transgenic mice overexpressing Glut12 improved peripheral insulin sensitivity, enhancing tissue-specific insulinstimulated glucose uptake (Purcell et al. 2011). In the same work, despite that a significant enhancement of the glucose uptake by EDL, soleus, fat and heart was observed in transgenic mice overexpressing Glut12, the heart showed the mildest improvement of all the tissues analyzed, while these transgenic mice exhibited a twofold increase in cardiac GLUT12 protein levels, one of the highest together with the brain (Purcell et al. 2011). A possible explanation to these observations was presented recently by Waller et al. (2013) showing that in contrast to what happens in other tissues (i.e. skeletal muscle and adipose tissue) Glut12 might be functioning mainly as a basal GLUT in the heart.

These evidences indicate that further in-depth studies on the physiological role of GLUT12 will make important contributions to better understand the connection between heart failure and pathologies impacting the glucose metabolism.

In this work, we used zebrafish (Danio rerio) model to investigate the in vivo function of glut12. Zebrafish is a genetically tractable model that offers unique advantages for in vivo studies as well as a valuable tool to study diseases impacting metabolism (Seth et al. 2013). We demonstrate that zebrafish glut12 structure and tissue distribution are highly conserved and similar to its mammalian ortholog. In addition, we show how glut12 in zebrafish is regulated by insulin and AMPK. Making use of reverse genetic approaches, we induced knockdown of glut12 in zebrafish. Our results indicate that glucose uptake mediated by glut12 is essential for cardiac development because glut12deficient embryos exhibited normal development but showed heart failure. Moreover, knockdown of glut12 led to insulin resistance and hyperglycemia being reminiscent of diabetic phenotypes. Taken together, our results indicate that glut12 is an essential GLUT in the heart where the reduction in glucose uptake due to glut12 deficiency leads to an impaired heart development. In addition, the metabolic features of the glut 12 morphants indicate that glut12 is an important insulin-regulated GLUT, making it a promising tool to assist in the development of drugs for the treatment of diabetic cardiomyopathies.

\section{Materials and methods}

\section{Zebrafish maintenance and lines}

Zebrafish were reared and handled in compliance with the local animal welfare legislation and maintained according to standard protocols (http://zfin.org). In this work, zebrafish lines used included WT AB/TL, Tg(myl7:eGFP) (Huang et al. 2003), Tg(fli1:GFP) (Lawson \& Weinstein 2002), and $T g(f a b p 10: d s R e d ; e l a 3 l: G F P)^{g z 12}$ (Farooq et al. 2008), hereafter named as 2-Color Liver Insulin Acinar Pancreas $(\operatorname{Tg}(2 C L I P))$. For heart extraction, insulin injections and imaging embryos were previously anesthetized in egg water containing $0.02 \%$ buffered 3 -aminobenzoic acid ethyl ester (Sigma).

\section{Sequence alignments and molecular phylogenetic analysis}

Multiple sequence alignments of the deduced amino acid sequence of the zebrafish Slc2a12 were performed using CLC Main Workbench 6.9.1 (CLC Bio A/S, Aarhus, Denmark, www.clcbio.com).

Phylogenetic relationships were inferred based on the deduced amino acid sequence for zebrafish Slc2a12, using the UPGMA method in CLC Main Workbench 6.9.1. For phylogenetic analysis, 5000 iterations were used to

Published by Bioscientifica Ltd. 
generate a bootstrap consensus tree. We used Genomicus genome browser (Louis et al. 2013) to perform the syntenic analysis of the genomic regions carrying glut12 loci in zebrafish compared with orthologs from other species of vertebrates.

\section{Morpholino design and injections}

To knockdown zebrafish glut12, two morpholino oligos (Gene Tools, LLC, Philomath, OR, USA) were designed, one of them blocking the translation of zebrafish glut12 (5'-TGCATCCATGTTCTTCAAGCGTTGT-3') and the other one targeting the splice acceptor site of exon 2 (5'-CCTTTCCCTAAATGACTAACCTGAT- $\left.{ }^{\prime}\right)$. Morpholinos were reconstituted in RNAse-free water, according to manufacturer's instructions. A standard control morpholino (5'-CCTCTTACCTCAGTTACAATTTATA-3') (Gene Tools, LLC) was injected at the same concentration as the glut12 MOs. MOs were diluted to a concentration of $0.5 \mathrm{mM}$ in $1 \times$ Danieau buffer $(58 \mathrm{mM} \mathrm{NaCl}$, $0.7 \mathrm{mM} \mathrm{KCl}, 0.4 \mathrm{mM} \mathrm{MgSO}_{4}, 0.6 \mathrm{mM} \mathrm{Ca}\left(\mathrm{NO}_{3}\right)_{2}, 5.0 \mathrm{mM}$ HEPES ( $\mathrm{pH} 7.6)$ ), and $1 \mathrm{nl}$ was injected at the one-cell stage using a Femtojet injector (Eppendorf, Hamburg, Germany).

\section{RNA isolation, CDNA synthesis, RT-PCR, and qPCR}

The embryos were collected at different stages $(24,48,72$, 96 , and $120 \mathrm{hpf}$ ), sampling 20 embryos per condition, using $1 \mathrm{ml}$ of QIAzol Lysis Reagent (Qiagen) and stored at $-80^{\circ} \mathrm{C}$ until its isolation. Also, to study the tissue distribution of slc2a12, different tissues from adult zebrafish (brain, eye, heart, intestine, liver, muscle, ovary, skin, spleen, and testis) were carefully extracted. The zebrafish were killed using an overdose of anesthesia (tricaine) and stored in RNAlater solution at $-80{ }^{\circ} \mathrm{C}$ until processed for RNA isolation. Total RNA was reverse transcripted into cDNA using IScript Reverse Transcriptase (Bio-Rad Laboratories B.V.). All samples were treated with RQ1 DNAse (Promega) to remove any residual genomic DNA. Conventional PCR was performed using DreamTaq Green DNA Polymerase (Thermo Scientific, Rockford, IL, USA), following manufacturer's indications. qPCR analysis was performed using iQ SYBR Green supermix (Bio-Rad Laboratories B.V.). The reactions were run in a iCycler Thermal Cycler (Bio-Rad Laboratories B.V.) using the following protocol: $3 \mathrm{~min}$ at $95^{\circ} \mathrm{C}$, followed by 40 cycles of $15 \mathrm{~s}$ denaturation at $95^{\circ} \mathrm{C}$ and $30 \mathrm{~s}$ at the corresponding melting temperatures, and a final melting curve of 81 cycles from $55^{\circ} \mathrm{C}$ to $95^{\circ} \mathrm{C}\left(0.5^{\circ} \mathrm{C}\right.$ increments every $\left.10 \mathrm{~s}\right)$.
mRNA expression levels were normalized against the expression of ppial as a housekeeping gene. The primer sequences used in this study are given in Supplementary Table 1, see section on supplementary data given at the end of this article.

\section{Purification of hearts from zebrafish embryos}

Extraction of hearts from 48 and $72 \mathrm{hpf}$ zebrafish embryos was accomplished as described previously (Burns \& MacRae 2006). A total of 150-200 hearts were used per sample. After extraction the hearts were kept in QIAzol Lysis Reagent (Qiagen) at $-80^{\circ} \mathrm{C}$ until its RNA isolation.

\section{Heartbeat rate and analysis}

To quantify the length of the cardiac cycle in zebrafish embryos and to study the differences between heartbeat rate in glut12 morphants and WT, a Hamamatsu C9300-221 high-speed CCD camera was used following the protocol described by Tessadori et al. (2012). To quantify heart rhythm, we drew kymographs for atrium or ventricle of WT and morphant embryos at 48 and $72 \mathrm{hpf}$. A total of 9-18 embryos were recorded for each condition and a total of 20 cardiac cycles were measured per embryo using ImageJ 64 (Rasband, W.S., ImageJ, U. S. National Institutes of Health, Bethesda, MD, USA, http://imagej.nih.gov/ij/, 1997-2014).

\section{Insulin and metformin administration and glucose measurements}

Insulin was injected into the zebrafish larvae as described previously (Marín-Juez et al. 2014b). For exposure to $250 \mu \mathrm{M}$ metformin, zebrafish embryos were dechorionated at $48 \mathrm{hpf}$ and treated with the compound for $48 \mathrm{~h}$ at $28^{\circ} \mathrm{C}$. Metformin was added to the water. Glucose measurements were performed using a fluorescence-based enzymatic detection kit (Biovision, Mountain View, CA, USA) as previously described (Jurczyk et al. 2011).

\section{Imaging}

Bright-field images were obtained using a Leica M165C stereomicroscope equipped with a DFC420C digital color camera (Leica Microsystems, Wetzlar, Germany). For fluorescent image acquisition, a Leica MZ16FA stereo fluorescence microscope equipped with a DFC420C digital color camera (Leica Microsystems) was used except in the case of fluorescent pictures of the valves, for which a Leica TCS SPE confocal laser scanning microscope

Published by Bioscientifica Ltd 
(Leica Microsystems) was used. Image analysis and Z-stacks projections were processed using Image 64 (Rasband, W.S., ImageJ, U. S. National Institutes of Health, Bethesda, MD, USA, http://imagej.nih.gov/ij/, 1997-2014).

\section{Transcriptome analysis}

The WT embryos were injected with $0.5 \mathrm{mM}$ of either standard control or splice glut12 MO. Ten larvae per sample were pooled and three samples per condition were generated for the analysis. Briefly, $1 \mu \mathrm{g}$ of RNA was isolated using TRIzol reagent (Life Technologies) and total RNA was extracted according to the manufacturer's instructions. RNA samples were treated with DNaseI (Life Technologies) to remove residual genomic DNA. RNA integrity was analyzed by Lab-on-a-chip analysis (Agilent, Amstelveen, The Netherlands). The average RIN value of the RNA samples was 9.7 with a minimum of 9.5. A total of $2 \mu \mathrm{g}$ of RNA was used to make RNA-seq libraries using the Illumina TruSeq RNA Sample Preparation Kit v2 (Illumina, Inc., San Diego, CA, USA). In the manufacturer's instructions, two modifications were made. In the adapter ligation step, $1 \mu \mathrm{l}$ (instead of $2.5 \mu \mathrm{l}$ ) adaptor was used. In the library size selection step, the library fragments were isolated using a double-Ampure XP purification with a $0.7 x$ beads to library ratio (Beckman Coulter, Woerden, The Netherlands). The resulting mRNA-seq library was sequenced using an Illumina HiSeq2500 instrument (Illumina, Inc.) according to the manufacturer's description with a read length of $2 \times 50$ nucleotides. Image analysis and base calling were done by the Illumina HCS version 2.0.12. Data analysis was performed using Genetiles software (www.genetiles.com, W J Veneman, J de Sonneville, KJ van der Kolk, A Ordas, Z Al-Ars, A H Meijer and H P Spaink 2014, unpublished observations). False discovery rate (FDR)-adjusted $P$ values were calculated based on the algorithm of Benjamini \& Hochberg (1995). The raw RNA-seq data have been deposited in the NCBI GEO database under accession number GSE59683. Gene ontology (GO) analysis was performed with DAVID Bioinformatics Resources 6.7 (Dennis et al. 2003).

\section{Statistical analyses}

Statistical differences were analyzed with Prism 6.0 (GraphPad Software, San Diego, CA, USA) using t-test for comparisons between two groups and one-way ANOVA (with Tukey's post hoc test correction) for multiple group comparisons and considered to be significant at $P<0.05$.

\section{Results}

\section{Characterization of zebrafish glut12 ortholog}

The zebrafish glut12 gene maps to chromosome 12 and shares a high similarity with human GLUT12 in its genomic sequence, both containing five exons (Supplementary Figure 3, see section on supplementary data given at the end of this article). Phylogenetic analysis to determine the conservation degree of the putative zebrafish Glut12 protein showed that it clustered with GLUT12 sequences from other vertebrates, being more closely related to human GLUT12 than to human GLUT10, another closely related Class 3 GLUT (Supplementary Figure 1A).

Comparison of the zebrafish Glut12-deduced amino acid sequence with those from other vertebrates showed a high percentage of sequence identity in all the cases (Supplementary Table 2, see section on supplementary data given at the end of this article and Supplementary Figure 1B). In addition, comparison of the genomic regions carrying the GLUT12 loci showed that the genes flanking zebrafish glut12 are syntenic across the different species analyzed, confirming its orthology among vertebrates (Supplementary Figure 2A).

Next, to address whether tissue expression of zebrafish glut12 is similar to that in humans using qPCR we analyzed the mRNA levels in a number of tissues extracted from adult zebrafish. This analysis revealed that, as in humans, zebrafish glut12 expression is widely distributed over various organs with the highest expression levels in skeletal and cardiac muscles (Supplementary Figure 2B). It has been shown by in situ hybridization that glut12 is expressed during the early development in the midbrain, eye, somites, and CNS (Thisse \& Thisse 2004, Tseng et al. 2009). As we failed to reliably detect glut12 expression in the embryonic heart by in situ hybridization, we extracted the hearts of zebrafish embryos at 48 and 72 hpf. RT-PCR analysis of the RNA extracted from these samples confirmed the expression of glut12 in the zebrafish heart at these stages (Supplementary Figure 2C).

\section{Insulin and metformin stimulate glut12 expression}

Human GLUT12 translocation to the plasma membrane in skeletal muscle is stimulated by insulin (Stuart et al. 2009). To better characterize the mechanisms regulating zebrafish glut12, we stimulated zebrafish larvae with insulin using a newly developed technique (Marín-Juez et al. 2014b). Zebrafish larvae at 4 days post fertilization (dpf) were injected into the caudal aorta with $100 \mathrm{nM}$ and

Published by Bioscientifica Ltd 
$1 \mu \mathrm{M}$ human recombinant insulin. qPCR analysis of glut12 expression was performed in zebrafish larvae at $0.5,1,2$, and $4 \mathrm{~h}$ post injection (hpi). Our results confirmed that injection of $100 \mathrm{nM}$ or $1 \mu \mathrm{M}$ insulin significantly stimulated glut12 expression at 0.5 and 4 hpi (Fig. 1A). In mammals, GLUT4 transcription is known to be stimulated
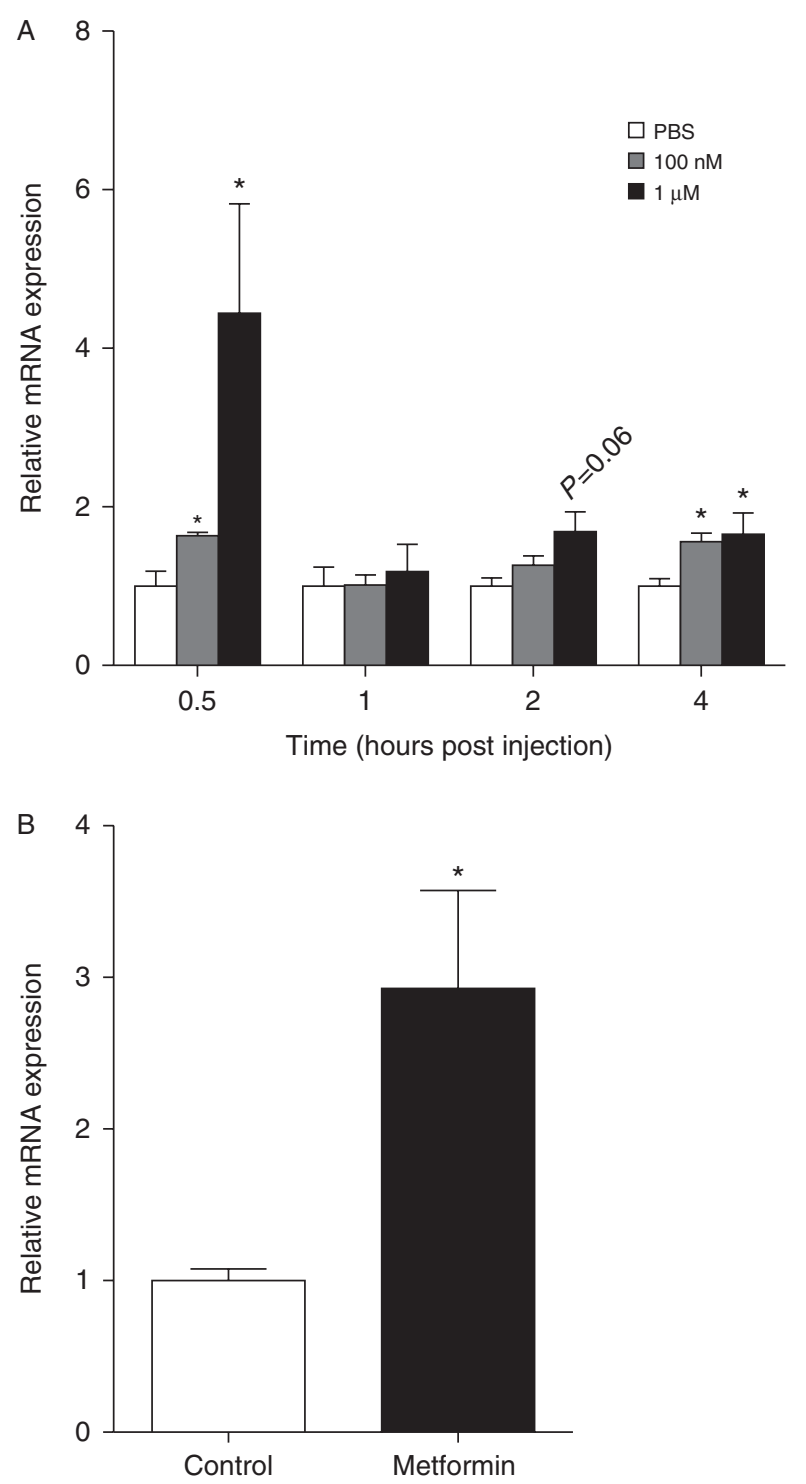

Figure 1

glut12 expression is stimulated by insulin and metformin. (A) Zebrafish larvae were injected with human recombinant insulin at $100 \mathrm{nM}$ and $1 \mu \mathrm{M}$ in the caudal aorta at $4 \mathrm{dpf}$. Control embryos were injected with PBS. RNA samples for QPCR analysis were taken at $0.5,1,2$, and $4 \mathrm{~h}$ after injection and glut12 mRNA levels were measured. (B) Relative glut12 expression after the exposure to metformin for $48 \mathrm{~h}$ at $250 \mathrm{mM}$. Data (mean \pm s.E.M.) are derived from a combination of four biological replicates ( $n=5$ larvae per group) and expressed relative to the control, which is set to 1 . *Significant differences between treatments $(P<0.05)$. via AMP-activated protein kinase (AMPK; Jorgensen et al. 2006). As glut4 is not present in zebrafish (Tseng et al. 2009, Marín-Juez et al. 2014a), we hypothesized that zebrafish glut12 may be functioning as the main insulin-regulated GLUT and therefore being regulated by similar mechanisms. To test this hypothesis, we stimulated zebrafish embryos with $250 \mu \mathrm{M}$ metformin, an indirect AMPK activator (Zhou et al. 2001, Viollet et al. 2012). qPCR analysis confirmed that glut12 expression was significantly stimulated after metformin administration, resembling observations of mammalian GLUT4 (Fig. 1B). These evidences suggest that zebrafish glut12 expression is regulated via insulin and AMPK signaling pathways, supporting the notion that in the absence of glut4, glut12 in zebrafish could be the main insulin-regulated GLUT.

\section{glut12 deficiency causes impaired cardiac development}

To further study its physiological role, we resorted to reverse genetic approaches to abrogate glut12. The knockdown was performed using an antisense morpholino designed to interfere with the splicing between exons 2 and 3 (splice MO) (Supplementary Figure 3A, see section on supplementary data given at the end of this article). To ensure the specificity of this morpholino, we also designed a translation-blocking morpholino (ATG MO) (Supplementary Figure 3A). The embryos injected with the splice MO developed normally with exception of the appearance of heart edema at $72 \mathrm{hpf}$ (Fig. 2A) and onwards (Supplementary Figure 3B). This was phenocopied by embryos injected with the ATG MO, confirming the specificity of the phenotype (Fig. 2A). To assess the extent of the spliceblocking activity of the splice MO, we carried out RT-PCR analysis of zebrafish embryos injected either with the control or with the splice MO from 24 to $120 \mathrm{hpf}$. Our results confirmed the efficiency of the morpholino showing the appearance of an aberrant splice product at all the different stages studied (Fig. 2B). In view of its efficiency and the possibility to easily confirm the knockdown, we chose to use the splice MO (hereafter named glut12 MO) for the rest of the study.

We then investigated whether the glut12 deficiency might be causing alterations in the heart development prior to the appearance of the cardiac edema. For this purpose, we injected the glut12 MO in $T g(m y l 7: e G F P)$ zebrafish, which allows in vivo visualization of myocardial cells. By 48 hpf, morphant embryos showed defects in chamber morphology, revealing that a large proportion of morphant embryos presented alterations in the loop formation exhibiting either abnormal (47\%) or no looping

Published by Bioscientifica Ltd 
A
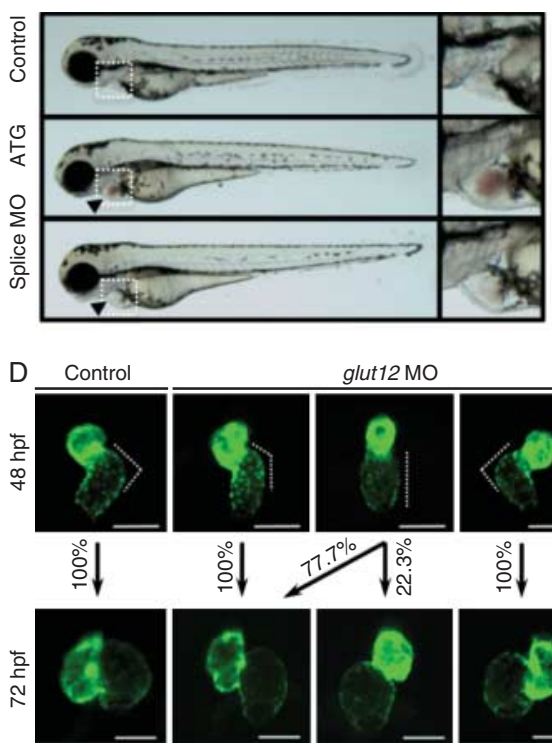
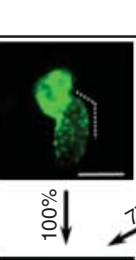

glut12 MO
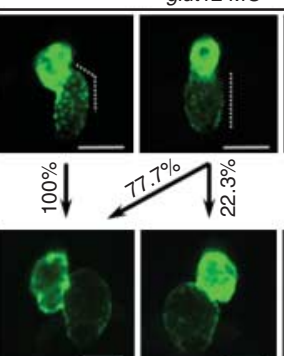
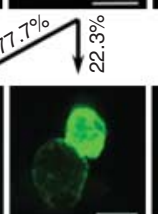

B

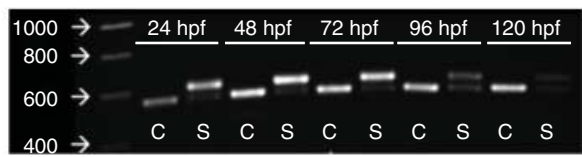

$E$

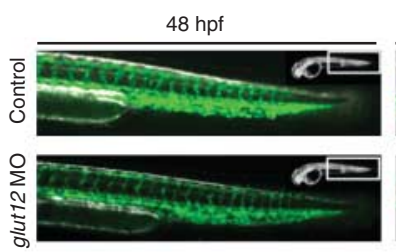

C
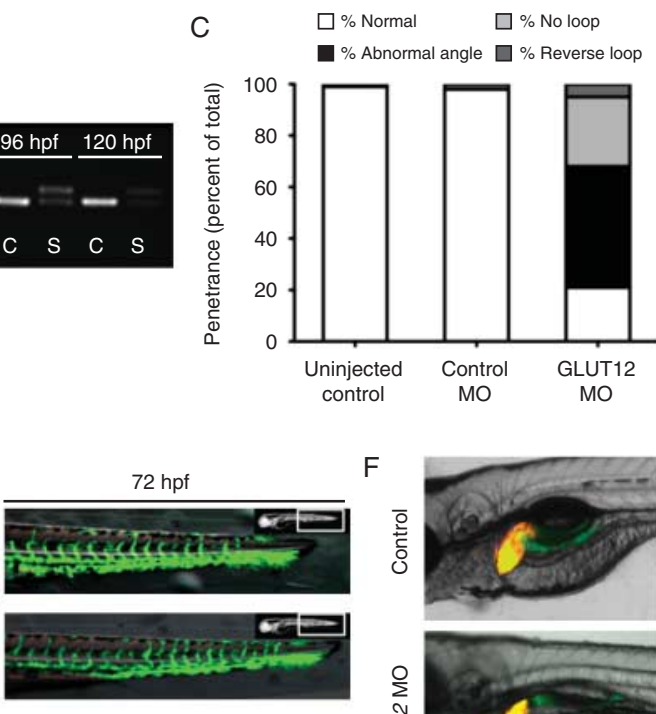

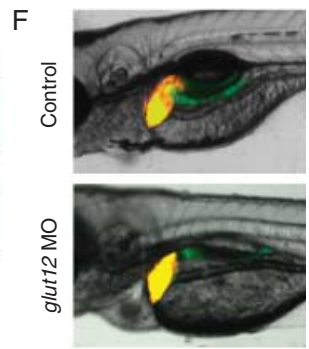

Figure 2

glut12 knockdown leads to impaired heart development. (A) Zebrafish embryos at $72 \mathrm{hpf}$ injected with either ATG or control MOs exhibited heart edema (black arrowhead). White dotted boxes indicate the regions shown in the inserts. (B) RT-PCR analysis of larvae from 24 to $120 \mathrm{hpf}$ injected with control and glut $12 \mathrm{MO}$ to assess the extent of the splice-blocking activity of the splice MO. (C) The penetrance of the g/ut12 knockdown was studied observing the different cardiac phenotypes present in the $T g$ (myl7:eGFP) embryos injected with the glut12 MO. Phenotypes were classified

(27\%) (Fig. 2C). In some cases reverse looping was observed, but despite that the incidence was slightly higher (5\%), this phenomenon could be observed also in control embryos (2\%) (Fig. 2C). The heart loop formation begins by approximately $36 \mathrm{hpf}$ and is complete by $48 \mathrm{hpf}$ (Bakkers 2011). This fact, together with the previous observations, led us to hypothesize that glut12 deficiency would cause a reduction in glucose uptake by the heart, resulting in a reduced growth and proliferation of cardiac cells impairing the development of the heart. In this regard, to confirm that the observed phenotype was not restored after $48 \mathrm{hpf}$, we injected $\operatorname{Tg}(m y l 7: e G F P)$ with the glut12 $\mathrm{MO}$ and followed larval heart development from 48 to $72 \mathrm{hpf}$. We observed that, while control and reversed loop hearts developed normally, hearts with abnormal looping angle at 48 and $72 \mathrm{hpf}$ fail to loop, as a consequence the cardiac chambers fail to rotate, exhibiting a phenotype more similar to $48 \mathrm{hpf}$ than to $72 \mathrm{hpf}$ (Fig. 2D). Interestingly, hearts without loop at $48 \mathrm{hpf}$ developed into the same phenotype as hearts with abnormal angle at $72 \mathrm{hpf}$, with a reduced percentage of them displaying the atrium in the reverse position (22.3\%) according to the looping. (D) Further characterization of these embryos was carried out and the morphology of these hearts was studied at $72 \mathrm{hpf}$. 35 hearts of each type were analyzed. Scale bar indicates $150 \mu \mathrm{m}$.

(E) $T g$ (fli1:GFP) embryos were injected with control and glut12 MO and vasculature of the tail region was visualized at 48 and $72 \mathrm{hpf}$. (F) Tg(2CLIP) embryos were injected with the control and glut12 MO. Liver (yelloworange) and pancreas (green) were visualized at $96 \mathrm{hpf}$. A full colour version of this figure is available via http://dx.doi.org/10.1530/JOE-14-0539.

(Fig. 2D). To exclude the possibility that this could be due to alterations in the vasculature we injected $T g(f l i 1: G F P)$ with the glut12 MO. We confirmed that the vascular system developed normally in morphant embryos from 24 to $72 \mathrm{hpf}$ (Supplementary Figures 3C and 2E). In addition, we injected $T g(2 C L I P)$ embryos with glut $12 \mathrm{MO}$, the results showing that the liver and the pancreas also developed normally (Fig. 2F). Overall, our results confirm that glut12 deficiency alters heart development leading to the formation of an underdeveloped cardiac structure.

\section{Loss of glut12 leads to alterations in atrial and ventricular contraction resulting in arrhythmias}

In view of the previous observations, we hypothesize that an underdeveloped heart may exhibit alterations in the heart rate and chamber contraction. To characterize these parameters, we analyzed the heart rhythm of glut12 morphants by high-speed video imaging, quantifying heart rate and fractional sorting as described by Tessadori et al. (2012). As we observed that atrial and ventricle contractions were coupled in both control and morphant

Published by Bioscientifica Ltd 


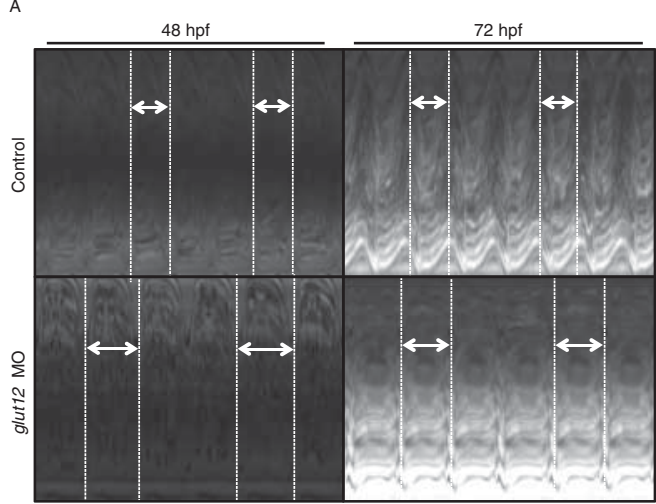

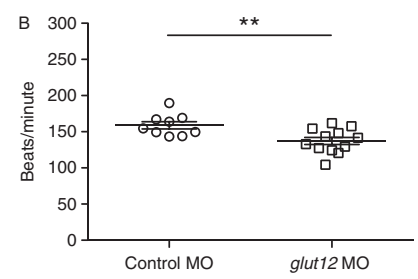

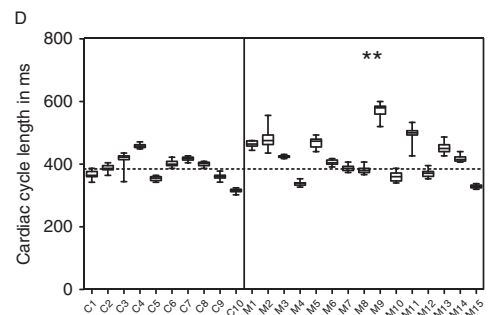

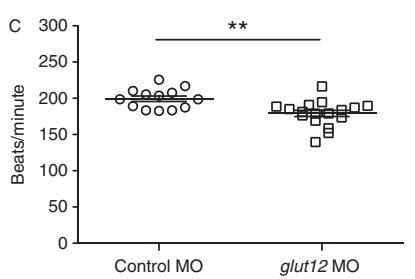

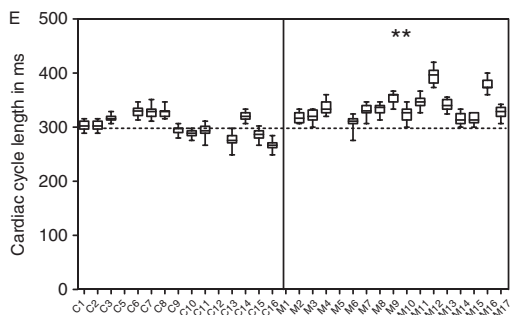

Figure 3

Cardiac contractility and rate are affected in glut12 deficient embryos. High-speed imaging to count heartbeats was used in glut12 morphants and control embryos. Embryonic hearts were imaged with a Hamamatsu C9300221 high-speed CCD camera at $150 \mathrm{fps}$ in a controlled temperature chamber $\left(28.5^{\circ} \mathrm{C}\right)$. Atrium kymographs from 48 and $72 \mathrm{hpf}$ embryos were used to compare the length of cardiac cycle (period) in controls and glut12 morphants. (A) The kymograph showed a longer period in morphants and was used to calculate the heart rate scatter dot plot representation of heartbeats per minute in $48 \mathrm{hpf}(\mathrm{B})$ and $72 \mathrm{hpf}(\mathrm{C})$ embryos. At least nine embryos per group were analyzed. Each dot represents one embryo.

$* * P<0.01$. Box-whisker plot representation of the cardiac cycle length of 20 successive heartbeats at $48 \mathrm{hpf}(\mathrm{D})$ and $72 \mathrm{hpf}(\mathrm{E})$. Every box-plot represents one embryo. $\mathrm{C}$, control; $\mathrm{M}$, glut12 morphants. ${ }^{*} P<0.01$. embryos (data not shown), we performed all the highspeed imaging-based measurements in the atrium of zebrafish embryos at 48 and $72 \mathrm{hpf}$. Heart rhythm was quantified by kymographs obtained from the high-speed videos. These kymographs showed that the time required for a full cardiac cycle in glut12 morphants was significantly extended (Fig. 3A). Next, based on these kymographs we quantified the cardiac cycle length and observed that the heart rate was significantly reduced (bradycardia) in glut 12 morphants at 48 and 72 hpf (Fig. 3B and C). In addition, cardiac length analysis confirmed the bradycardia and revealed irregular heart rhythm in glut12 morphants that could be observed in some cases at $48 \mathrm{hpf}$ (Fig. 3D) and clearly at $72 \mathrm{hpf}$ (Fig. 3E). These observations indicate that the delayed cardiac development caused by the deficiency in glut12 leads to heart failure.

\section{glut12 morphants exhibit blood toggling between heart chambers as a consequence of abnormal valve formation}

Low heart rates have been described to lead to abnormal valve formation (Vermot et al. 2009). To investigate this effect, we analyzed high-speed imaging videos of control and glut 12 morphant embryos at $72 \mathrm{hpf}$, because at this stage valves should be fully formed preventing any retrograde flow fraction (RTT; Vermot et al. 2009). Interestingly, we observed that while in control embryos blood flow was unidirectional following atrial and ventricular contractions, morphant embryos exhibited blood toggling between the atrial systole and the ventricular diastole (Fig. 4A, Supplementary Movies 1 and 2 , see section on supplementary data given at the end of this article). To confirm whether the alterations in heart rate affected valve morphogenesis in glut12 morphants causing blood toggling, we used the $T g(f l i 1: G F P)$ line to visualize the valve formation at $72 \mathrm{hpf}$. While in control embryos we could observe the valves in between both cardiac chambers, these were absent in glut12 morphants (Fig. 4B). Vermot et al. (2009) demonstrated that alterations in the heart rate cause valve dysgenesis via downregulation of klf2a. To confirm this, we carried out qPCR analysis in hearts extracted from control and glut12 morphant embryos at $72 \mathrm{hpf}$. Our results confirmed that $k l f 2 a$ expression was significantly down-regulated in morphant embryos (Fig. 4C). Taken together, these data indicate that the alterations in the heart rate as a consequence of the glut12 deficiency down-regulate klf2a leading to abnormal valve formation.

\section{Abrogation of glut12 leads to hyperglycemia and insulin resistance}

We set out to further investigate the physiological consequences of glut 12 abrogation. In view of the apparent

Published by Bioscientifica Ltd. 
A 1st atrial diastole
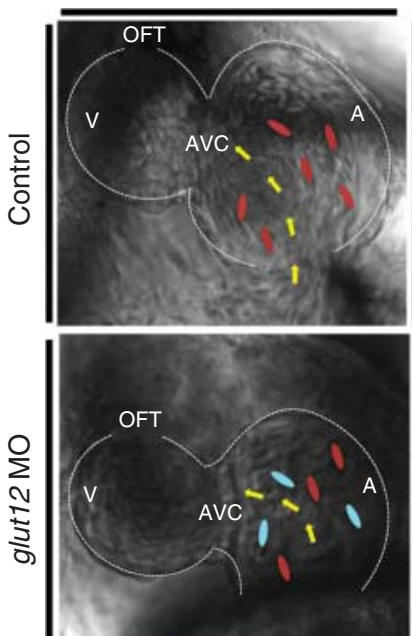

$\mathrm{B}$
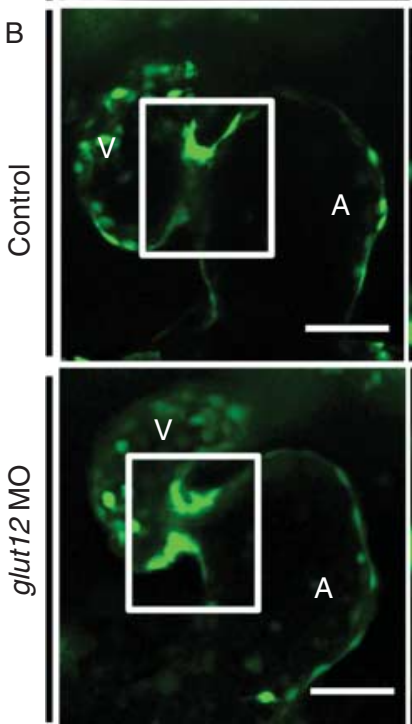

Atrial systole
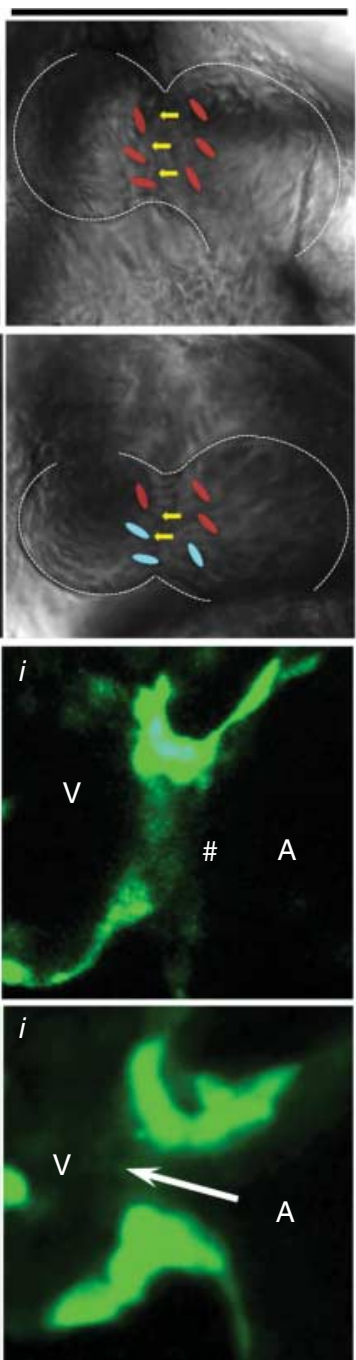

Ventricular diastole/systole
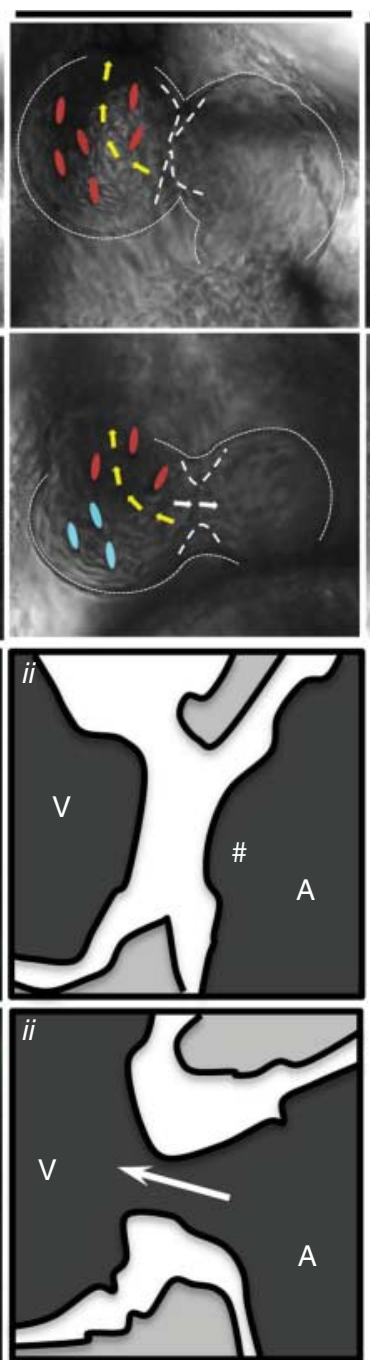

2nd atrial diastole
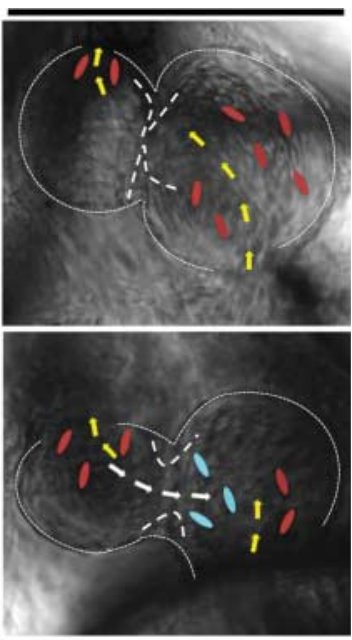

C

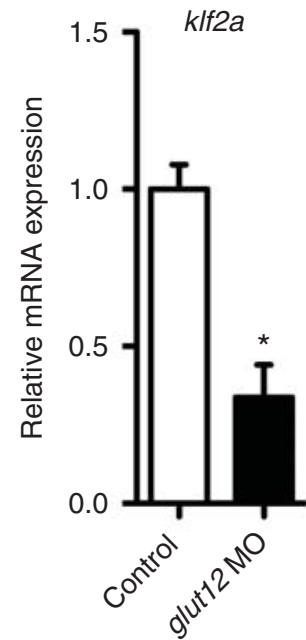

\section{Figure 4}

glut12 morphants exhibit backflows of blood as a consequence of abnormal valve formation. (A) Bright field images recorded by high-speed imaging comparing control and glut12 morphant embryos showing a schematic representation of a cardiac cycle in zebrafish embryos. A zebrafish embryonic heart at $72 \mathrm{hpf}$ is highlighted in white dotted contour and valves are highlighted in white dashed contour. Red ellipses, erythrocytes following normal flow; yellow arrows, normal blood flow blue ellipses, erythrocytes fraction returning to the atrium; white arrows, blood backflow (For more detailed information see Supplementary Movies 1 and 2). (B) To better visualize the valve morphology in morphant embryos compared to the control we used $T g(f l i 1: G F P)$. White boxes indicate the regions magnified in the sections $i$ and $i i$. Scale bar indicates $50 \mu \mathrm{m}$. (i) High magnifications of the AVC regions. \# indicates the closure of the AVC canal by the valves. The white arrow indicates the abnormal valve morphology and the consequent opening of the AVC. (ii) Schematic representation of the AVC regions. (C) Expression of klf2a was analyzed by qPCR. RNA was extracted from control and glut12 morphants at $72 \mathrm{hpf}$. Data (mean \pm S.E.M.) are combined from 4 biological replicates ( $n=150-200$ hearts per group) and expressed relative to control, which is set to $1 .{ }^{*} P<0.05$. $\mathrm{V}$, ventricle; $\mathrm{A}$, atrium; AVC, atrio-ventricular canal; OFT, outflow tract. A full colour version of this figure is available via http://dx.doi.org/10.1530/ JOE-14-0539.

In agreement with previous observations, free glucose levels appeared to diminish after $2 \mathrm{dpf}$, increasing again from 3 to $5 \mathrm{dpf}$ (Jurczyk et al. 2011, Gut et al. 2013). This could correspond to an initial increase in glucose levels that might drive the maturation of beta pancreatic cells as observed in mammals (Hellerstrom \& Swenne 1991, http://joe.endocrinology-journals.org DOI: 10.1530/JOE-14-0539
(C) 2015 Society for Endocrinology Printed in Great Britain 
Cao et al. 2004), dropping at 3dpf and increasing afterwards, coinciding with the functional onset of the hepatic gluconeogenesis (Gut et al. 2013). Our results showed that between 3 and $5 \mathrm{dpf}$, glut12-deficient embryos presented significantly higher free glucose levels (Fig. 5A). Next, to investigate whether the knockdown of glut12 would be translated into a reduced insulin sensitivity, we measured free glucose levels in $4 \mathrm{dpf}$ larvae at $15,30,60$, and $120 \mathrm{~min}$ post injection of $1 \mathrm{nl}$ of $100 \mathrm{nM}$ human insulin. Interestingly, in glut12-morphant larvae, insulin administration caused no significant reduction in free glucose levels, in contrast to control embryos where the expected hypoglycemic effect was observed

A
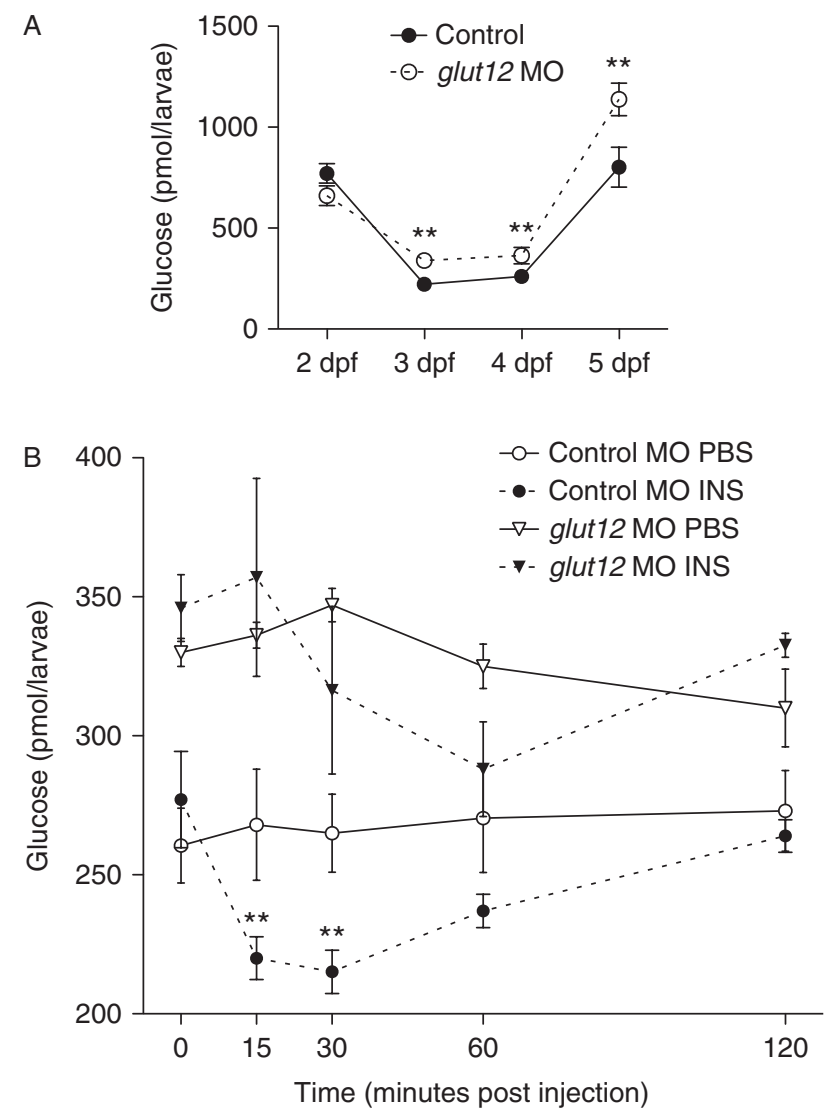

Figure 5

Knockdown of glut12 leads to hyperglycemia and insulin resistance. (A) Glucose dynamics of glut12 morphants were studied and compared to control embryos. Samples for free glucose measurements were taken at 2 , 3,4 and 5 dpf. Data (mean \pm s.E.M.) are combined from five biological replicates ( $n=10$ embryos per group). ${ }^{*} P<0.01$. (B) Control and g/ut12 morphant larvae were injected with human recombinant insulin at $100 \mathrm{nM}$ in the caudal aorta at $4 \mathrm{dpf}$. In each case PBS-injected larvae were used as control. Samples for glucose measurements were taken at $0,15,30,45,60$ and $120 \mathrm{~min}$ after injection. Data (mean \pm s.E.M.) are combined from five biological replicates ( $n=10$ embryos per group). ${ }^{*} P<0.01$.
(Fig. 5B and Supplementary Figure 4, see section on supplementary data given at the end of this article).

Altogether, these data suggest that glut12 abrogation causes a reduction in glut12-mediated glucose uptake causing hyperglycemia and insulin resistance, resembling a diabetic phenotype.

\section{Transcriptomic profile confirms the observed cardiac and diabetic phenotype and reveals tissue-specific effects upon glut12 knockdown}

To better understand the impact of glut12 deficiency at the whole embryo level, we carried out an RNAseq-based transcriptome analysis of $4 \mathrm{dpf}$ glut12-morphant larvae compared with control larvae. To analyze the data, we set significance cut-offs at 1.5 -fold change at $P<0.05$ (FDR adjusted $P$ value). In this way, we obtained 1099 upregulated genes and 290 down-regulated genes. Next, to further characterize the differentially expressed genes (DEGs), we performed a GO analysis for functional classification (Supplementary Tables 3, 4, and 5, see section on supplementary data given at the end of this article). In addition, RNA-seq data were validated by qPCR, observing the same effects on the expression of the genes analyzed by both techniques (Supplementary Figure 5, see section on supplementary data given at the end of this article).

The study of GO terms showed that glut12 deficiency altered biological processes involved in the development of the main organs responsible for glycemic control, which are commonly affected in diabetes (e.g., heart, liver, kidney, and skeletal muscle). In addition, other categories involving important pathways related to insulin signaling and action appeared dysregulated, such as the JAK-STAT and protein kinase cascades. When looking at cellular component and molecular function GO terms, we found an enrichment in categories involved in neural development. For more detailed analysis of the transcriptomic profile of the glut12 morphants, we selected genes important for cardiac, hepatic, and neural function and development and insulin signaling pathway, as well as genes involved in metabolism and diabetes and/or insulin resistance (Fig. 6). Among DEGs involved in the insulin signaling pathway, a number of genes important to this pathway appeared up-regulated (insr, $i r s 2, p i k 3 c a, p d k 2)$. Moreover, we observed a number of DEGs known to be important for the function and development of the heart (klf2a, actc1a, gata4, srf, snx10a, tcap, dbh, tek), liver (mtf1, stat3, rgs3, ppar6) and brain (map1a, gamt, nos1, gnrh2, tph1a, gpr3, ddita). Notably, we found that several genes known to be dysregulated in

Published by Bioscientifica Ltd. 


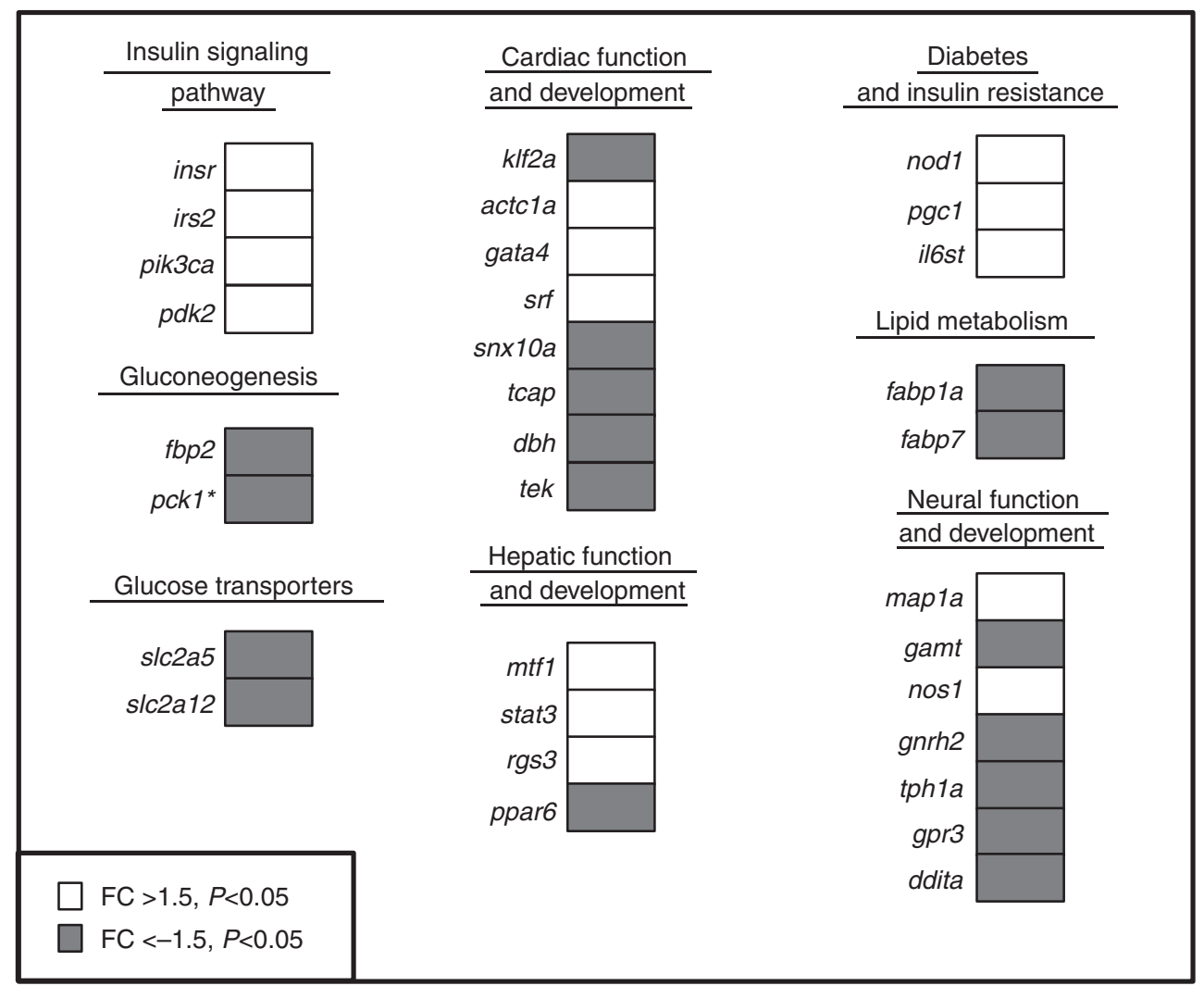

Figure 6

Transcriptomic profile of glut12-deficient larvae. Transcriptomic analysis was performed comparing control and glut 12 morphants at $4 \mathrm{dpf}$. Grey boxes represent down-regulation and white boxes represent up-regulation. Significance cut-offs were set at fold change $(F C)>1.5$

diabetes or in insulin resistance (nod1, pgc1, il6st) were up-regulated in glut12 morphants. Furthermore, genes involved in lipid metabolism (fabp $1 a, f a b p 7)$ and gluconeogenesis $(f b p 2, p c k 1)$ were down-regulated in glut12-deficient larvae. Finally, we found two GLUTs down-regulated glut5 and glut12, the latter confirming the efficiency of the splice-blocking morpholino.

\section{Discussion}

Despite the fact that the heart can use a broad range of substrates as fuel, its metabolic machinery is finely regulated to ensure an optimal usage of the energy provided by these substrates. Aberrant metabolism is a common result of heart failure, for which cardiomyocytes resort to the usage of different substrates to fulfill the energetic needs of this metabolically demanding organ (van der Vusse et al. 2000, van Bilsen et al. 2004). Therefore, pathologies altering the control of metabolism (e.g., diabetes and insulin resistance) have critical and $P<0.05$. *Due to its relevance mediating a rate-limiting step of gluconeogenesis, $p c k 1$ has been included in the figure despite its FC being 1.42 with a $P<0.05$.

consequences contributing to the development and worsening of cardiomyopathies (Bell 2003b). One of the most recently discovered GLUTs, GLUT12, has been shown to be regulated by insulin in skeletal muscle (Stuart et al. 2009) and importantly has been described as a basal cardiac GLUT (Waller et al. 2013). Therefore, to gain insights into the physiological role of this GLUT, in this work we set out to study the functional consequences of glut12 abrogation. As a result we could show how glut12 abrogation impairs cardiac development, altering the contraction rate that in turns leads to alterations in the valvulogenesis. Moreover, we observed that glut12deficient larvae were insulin resistant, exhibiting reduced insulin sensitivity and hyperglycemia, features characteristic of the diabetic phenotypes.

Both GLUT4 and GLUT12 have been shown to perform an important role in the cardiac muscle. Studies using a canine model for chronic heart failure showed that the expression of both GLUT4 and GLUT12 is affected in specific myocardial structures (Ware et al. 2011).

Published by Bioscientifica Ltd 
Moreover, Glut4 deficiency in the heart has been reported to cause cardiomyopathies (Domenighetti et al. 2010). Supporting this notion, our data clearly show that the knockdown of glut12 impairs embryonic heart development leading to alterations in contractility and bradycardia. Embryonic heart development is a tightly regulated process for which the energy source has been found to be a key determinant. During early development, cardiomyocyte precursors rely mainly on glycolysis to obtain energy (Chung et al. 2007, 2010). In addition, it has been described that proper heart formation is the result of a combination of genetic and mechanical factors that result in the proper development of the cardiac structures (Hove et al. 2003, Vermot et al. 2009). Valvulogenesis appears to be especially sensitive to alterations in this mechanogenetic equilibrium. Recently, Vermot et al. (2009) demonstrated that alterations in the RTT result in defective valve formation. Moreover, in the same study it was shown that variations in the heart rate lead to variations in the RTT via down-regulation of $k l f 2 a$, causing valve dysgenesis as a consequence (Vermot et al. 2009). KLF2's responsiveness to the blood flow is well conserved and it has been found expressed in regions subjected to high shear forces in human, chicken, and zebrafish hearts (e.g., the site of valve formation) (Dekker et al. 2002, Groenendijk et al. 2004, Vermot et al. 2009). As in mammals, glut 12 is found highly expressed in the heart of zebrafish and its abrogation resulted in clear alterations of the heart development probably as a consequence of the energy imbalance experienced by the cardiac muscle due to deficient glucose uptake. In view of this, we propose that the deficiency in glucose uptake caused by glut 12 abrogation impaired the embryonic heart development altering the loop formation, the contractility of myocardial cells, and the heart rate. This, in turn, altered the shear forces in the cardiac endothelium causing klf2a down-regulation and as a consequence valve dysgenesis (Fig. 7). Moreover, our deep sequencing data confirmed the down-regulation of $k l f 2 a$ and showed that the expression of a number of genes important for cardiac functioning and development was affected. Interestingly, gata4 appeared up-regulated in our data set. Gata4 overexpression has been reported to cause cardiac hypertrophy (Liang et al. 2001). During physiological cardiac

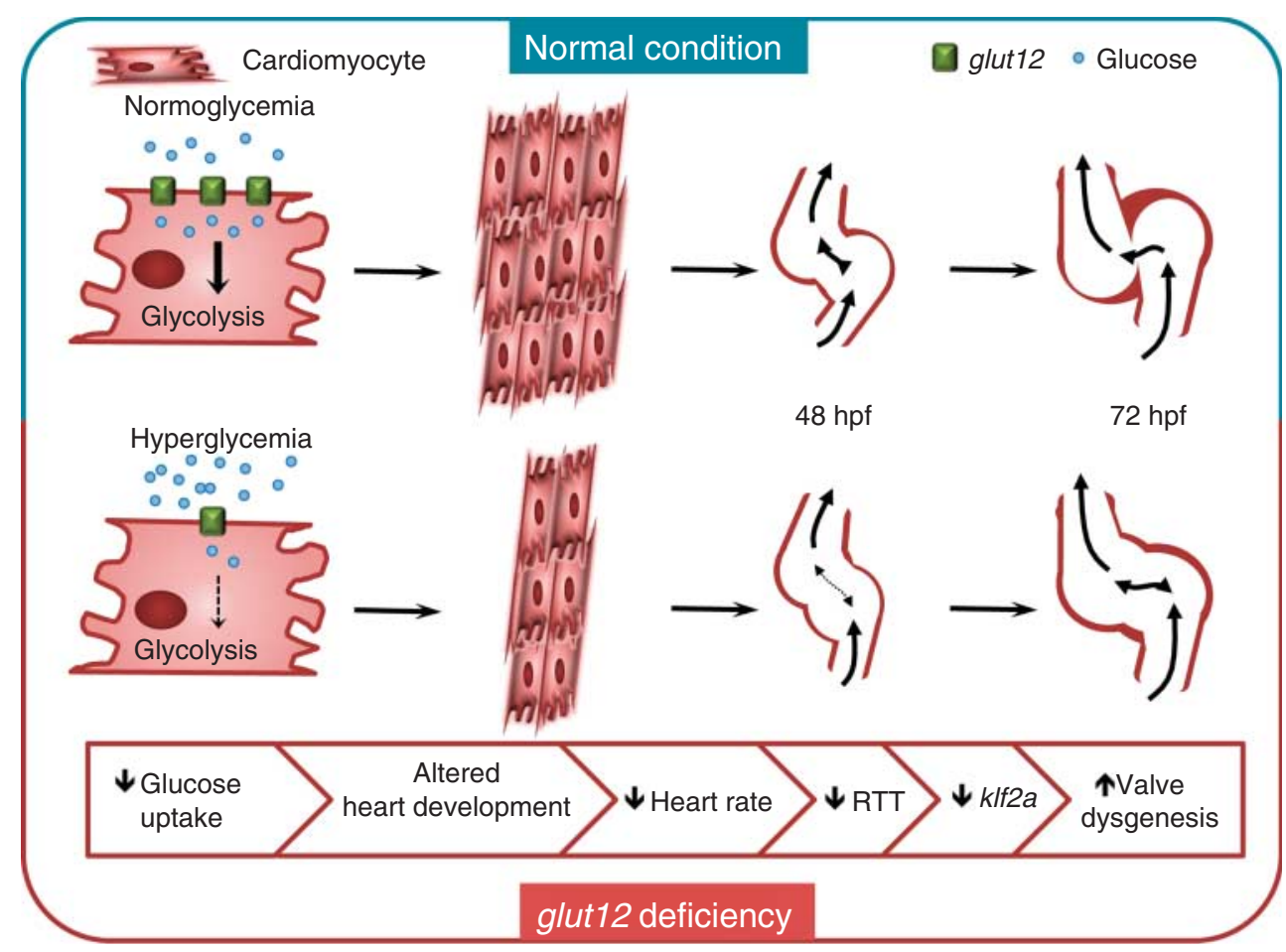

\section{Figure 7}

Proposed model for cardiac glut12 deficiency. Cardiac glucose uptake is reduced as a consequence of the glut12 abrogation. This impact on energy balance impairs the cardiac development altering the contractility of the myocardial cells and the heart rate. Embryonic hearts exhibiting poor
(๑) 2015 Society for Endocrinology Printed in Great Britain looping and bradycardia have a reduced RTT down-regulating klf2a, which in turn causes valve dysgenesis. A full colour version of this figure is available via http://dx.doi.org/10.1530/JOE-14-0539. 
hypertrophy, cardiac metabolism switches from glycolysis to fatty acid $\beta$-oxidation as the main source of energy (Lorell \& Grossman 1987). However, in adult hearts experiencing pathophysiological hypertrophy, cardiac metabolism reverts to glycolytic metabolism resembling the fetal scenario (Lorell \& Grossman 1987, Depre et al. 1998, Razeghi et al. 2001). In our case, gata4 up-regulation in glut12 morphants could reflect an attempt to switch to a less glucose-dependent cardiac metabolism. In our opinion, a scenario where gata 4 up-regulation may be reflecting pathological hypertrophy is most likely. The observed down-regulation in glut12 morphants of snx10a, $d b h$, and tek supports this idea, because lack or deficiency of these genes has been reported to alter cardiac function, in some cases leading to heart failure (Swoap et al. 2004, Tachibana et al. 2005, Chen et al. 2012). We performed deep sequencing analysis at whole-embryo level to assess the global impact of glut12 deficiency. Future transcriptomic studies focused on the heart in a tissue-specific manner will be needed to further study the observed phenotype.

GLUT12 has been proposed to be a secondary GLUT that complements GLUT4 mediating, insulin-stimulated glucose uptake (Stuart et al. 2009, Purcell et al. 2011). It has been shown that, in skeletal muscle, GLUT12 translocates to the plasma membrane together with GLUT4 after insulin stimulation (Stuart et al. 2009). These observations could also explain why Glut4-knockout mice still showed insulin-stimulated glucose uptake and did not develop hyperglycemia (Katz et al. 1995, Stenbit et al. 1996). Moreover, Stenbit et al. showed later that Glut4 heterozygote knockout mice displayed reduced insulin sensitivity in the skeletal muscle, hyperinsulinemia, and hyperglycemia (Stenbit et al. 1997). In this work, the authors propose that, while heterozygous mice have Glut4 until the age of 8 weeks and then experience a decrease in Glut4 content, Glut4-null mice have complete lack of the transporter developing and activating other mechanisms to compensate for this deficiency (Stenbit et al. 1997). One of these mechanisms could be Glut12.

Current data support the notion that in zebrafish glut4 has been lost (Tseng et al. 2009, Marín-Juez et al. 2014a), making it an attractive model for the study of glut12, which could be functioning as the main insulinregulated GLUT. Zebrafish glut12 presents a high degree of conservation compared with its human counterpart in its protein structure, syntenic distribution, and tissue expression pattern. Moreover, our data also show that, in agreement with the observations in mammals, its expression is regulated by insulin. Recently, it has been published by our group that human insulin is effective in zebrafish larvae (Marín-Juez et al. 2014b). In that work, we showed how $4 \mathrm{dpf}$ larvae injected with $100 \mathrm{nM}$ insulin experienced transient hypoglycemia between 15 and 45 min post injection, becoming insulin resistant at 4 hpi (Marín-Juez et al. 2014b). Interestingly, the expression of glut12 is stimulated at 0.5 and $4 \mathrm{hpi}$, times at which zebrafish larvae are insulin sensitive and resistant respectively. The fast response observed at $0.5 \mathrm{hpi}$ could indicate that glut12 expression is rapidly stimulated in the presence of insulin to ensure an efficient glucose uptake. The latter stimulation could be explained as an attempt to compensate for the hyperglycemia that these larvae experience as a consequence of the hyperinsulinemia-induced insulin resistance that they experience at 4 hpi (Marín-Juez et al. 2014b).

As in mammals GLUT4 is known to be regulated by AMPK, we hypothesized that this could be the case for glut12, assuming that in zebrafish it is functioning as the main insulin-regulated GLUT in the absence of glut4. Our data showed that stimulation with the antidiabetic drug metformin, an indirect AMPK activator (Viollet et al. 2012), stimulates glut12 expression, suggesting that like mammalian GLUT4, zebrafish glut12 could be regulated via the AMPK signaling pathway. It is worth mentioning that because one of the main targets of metformin is the mitochondrial respiratory chain, it has been proposed that the activation of AMPK is a consequence of the energy imbalance that the cells experience when exposed to the drug (Viollet et al. 2012). In view of this, we cannot rule out the possibility that the observed effects might be due to AMPK-independent mechanisms. Future in-depth studies will help to elucidate this.

Interestingly, we show that glut12 deficiency caused hyperglycemia and insulin resistance. These results are in agreement with recent data showing that mice overexpressing Glut12 presented an improved insulin sensitivity (Purcell et al. 2011) and further support that in zebrafish glut12 is an insulin regulated GLUT. In addition, the observed hyperglycemia might be a consequence of the reduced glucose uptake by tissues expressing glut12, especially the skeletal muscle since this represents a high portion of the larvae body mass.

The transcriptomic profiling showed that nod1, il6st, srf and pgc1 were up-regulated in glut12 morphants supporting the observed phenotype since activation in the case of Nod1 or increased levels in the case of Il6st have been linked to insulin resistant states (Zuliani et al. 2010, Schertzer et al. 2011). Overexpression of $s r f$ is increased in

Published by Bioscientifica Ltd 
T2D but also has been reported to cause cardiomyopathy (Zhang et al. 2001) and Pgc1 $\alpha$ dysregulation has been extensively studied and linked to T2D. However, in the case of $P g c 1 \alpha$, the observations differ in a tissue specific manner, observing Pgc1 $\alpha$ up-regulation in the liver of mouse models for T1D and T2D (Puigserver \& Spiegelman 2003), and down-regulation in muscle from humans with T2D (Mootha et al. 2003, Patti et al. 2003). Moreover, Pgc1 $\alpha$ also plays a key role as a mediator of cardiac metabolism (Lehman et al. 2000, Arany et al. 2005). As noted before, future tissue specific studies will be helpful to elucidate the specific role of $p g c 1$ in a situation of glut12 deficiency.

It is worth mentioning that our transcriptomic analysis also showed enrichment in DEGs involved in neural function and development. Interestingly, Pujol-Gimenez et al. (2014) found that GLUT12 expression was increased in the frontal cortex of patients with Alzheimer disease, suggesting for the first time that GLUT12 might play a role in the development of Alzheimer disease.

In summary, in the present manuscript we demonstrate that glut12 deficiency causes heart failure and diabetic phenotypes during embryonic development. In addition, it has been previously hypothesized that during early embryonic development, Glut12 might be the main GLUT responsible for insulin-regulated glucose uptake (Macheda et al. 2002) since Glut4 is not expressed during early development, being only detectable at very low levels at late fetal/early postnatal life in mouse (Santalucia et al. 1992). In this regard, the zebrafish model for glut12 deficiency that we used in this study will be a unique tool to not only study fetal diabetic cardiomyopathies but also contribute to the development and discovery of new anti-diabetic drugs thanks to its characteristics as a vertebrate model with high-throughput screening possibilities.

\section{Supplementary data}

This is linked to the online version of the paper at http://dx.doi.org/10.1530/ JOE-14-0539.

\section{Declaration of interest}

The authors declare that there is no conflict of interest that could be perceived as prejudicing the impartiality of the research reported.

\section{Funding}

R M-J was supported by a Marie Curie fellowship as Experienced Researcher in the EU Initial Training Network FishForPharma (PITN-GA-2011-289209).

\section{Author contribution statement}

V J-A designed and performed experiments, analyzed the data, and wrote the paper. S J-R designed and performed experiments. J B participated in the design of the study, performed the statistical analysis, and made available facilities. H P S supervised the work, wrote the paper, and made available facilities and resources. $\mathrm{R} \mathrm{M}-\mathrm{J}$ conceived the study, designed and performed experiments, supervised the work, and wrote the paper. All authors assisted in editing of the manuscript and approved the final version.

\section{Acknowledgements}

The authors thank Silja B Burkhard for her assistance with the high-speed imaging and analysis; Sebastiaan A Brittijn for his assistance with the tissue extraction; Davy de Witt, Ulrike Nehrdich, and Laura van Hulst for fish caretaking. They gratefully thank Dr Didier $Y \mathrm{R}$ Stainier and Dr Bernard Peers for the $T g(2 C L I P)$ line and Dr Ron Dirks, Dr Hans Jansen, Hulya Ozupek, and Nynke Tuinhof-Koelma (ZF-screens B.V.) for assistance with RNA-seq analysis. They also want to thank to Wouter J Veneman, Dr Jan de Sonneville, and Kees-Jan van der Kolk for making the Genetiles software available before publication.

\section{References}

Arany Z, He H, Lin J, Hoyer K, Handschin C, Toka O, Ahmad F, Matsui T, Chin S, Wu PH et al. 2005 Transcriptional coactivator PGC- $1 \alpha$ controls the energy state and contractile function of cardiac muscle. Cell Metabolism 1 259-271. (doi:10.1016/j.cmet.2005.03.002)

Bakkers J 2011 Zebrafish as a model to study cardiac development and human cardiac disease. Cardiovascular Research 91 279-288. (doi:10.1093/cvr/cvr098)

Bell DS 2003a Diabetic cardiomyopathy. Diabetes Care 26 2949-2951. (doi:10.2337/diacare.26.10.2949)

Bell DS 2003 $b$ Heart failure: the frequent, forgotten, and often fatal complication of diabetes. Diabetes Care 26 2433-2441. (doi:10.2337/ diacare.26.8.2433)

Benjamini Y \& Hochberg Y 1995 Controlling the false discovery rate - a practical and powerful approach to multiple testing. Journal of the Royal Statistical Society. Series B (Methodological) 57 289-300.

van Bilsen M, Smeets PJ, Gilde AJ \& van der Vusse GJ 2004 Metabolic remodelling of the failing heart: the cardiac burn-out syndrome? Cardiovascular Research 61 218-226. (doi:10.1016/j.cardiores.2003.11.014)

Burns CG \& MacRae CA 2006 Purification of hearts from zebrafish embryos. BioTechniques 40 274, 276, 278 passim. (doi:10.2144/000112135)

Cao LZ, Tang DQ, Horb ME, Li SW \& Yang LJ 2004 High glucose is necessary for complete maturation of Pdx1-VP16-expressing hepatic cells into functional insulin-producing cells. Diabetes 53 3168-3178. (doi:10.2337/diabetes.53.12.3168)

Chen Y, Wu B, Xu L, Li H, Xia J, Yin W, Li Z, Shi D, Li S, Lin S et al. 2012 A SNX10/V-ATPase pathway regulates ciliogenesis in vitro and in vivo. Cell Research 22 333-345. (doi:10.1038/cr.2011.134)

Chung S, Dzeja PP, Faustino RS, Perez-Terzic C, Behfar A \& Terzic A 2007 Mitochondrial oxidative metabolism is required for the cardiac differentiation of stem cells. Nature Clinical Practice. Cardiovascular Medicine 4(Suppl 1) S60-S67. (doi:10.1038/ncpcardio0766)

Chung S, Arrell DK, Faustino RS, Terzic A \& Dzeja PP 2010 Glycolytic network restructuring integral to the energetics of embryonic stem cell cardiac differentiation. Journal of Molecular and Cellular Cardiology 48 725-734. (doi:10.1016/j.yjmcc.2009.12.014)

Dekker RJ, van Soest S, Fontijn RD, Salamanca S, de Groot PG, VanBavel E, Pannekoek H \& Horrevoets AJ 2002 Prolonged fluid shear stress induces a distinct set of endothelial cell genes, most specifically lung Kruppel-like factor (KLF2). Blood 100 1689-1698. (doi:10.1182/blood-2002-01-0046) http://joe.endocrinology-journals.org DOI: 10.1530/JOE-14-0539
() 2015 Society for Endocrinology Printed in Great Britain 
Dennis G Jr, Sherman BT, Hosack DA, Yang J, Gao W, Lane HC \& Lempicki RA 2003 DAVID: Database for Annotation, Visualization, and Integrated Discovery. Genome Biology 4 P3. (doi:10.1186/gb-2003-4-5-p3)

Depre C, Shipley GL, Chen W, Han Q, Doenst T, Moore ML, Stepkowski S, Davies PJ \& Taegtmeyer H 1998 Unloaded heart in vivo replicates fetal gene expression of cardiac hypertrophy. Nature Medicine 4 1269-1275. (doi:10.1038/3253)

Domenighetti AA, Danes VR, Curl CL, Favaloro JM, Proietto J \& Delbridge LM 2010 Targeted GLUT-4 deficiency in the heart induces cardiomyocyte hypertrophy and impaired contractility linked with $\mathrm{Ca}(2+)$ and proton flux dysregulation. Journal of Molecular and Cellular Cardiology 48 663-672. (doi:10.1016/j.yjmcc.2009.11.017)

Farooq M, Sulochana KN, Pan X, To J, Sheng D, Gong Z \& Ge R 2008 Histone deacetylase 3 (hdac3) is specifically required for liver development in zebrafish. Developmental Biology 317 336-353. (doi:10.1016/j.ydbio.2008.02.034)

Groenendijk BC, Hierck BP, Gittenberger-De Groot AC \& Poelmann RE 2004 Development-related changes in the expression of shear stress responsive genes KLF-2, ET-1, and NOS-3 in the developing cardiovascular system of chicken embryos. Developmental Dynamics 230 57-68. (doi:10.1002/dvdy.20029)

Gut P, Baeza-Raja B, Andersson O, Hasenkamp L, Hsiao J, Hesselson D, Akassoglou K, Verdin E, Hirschey MD \& Stainier DY 2013 Wholeorganism screening for gluconeogenesis identifies activators of fasting metabolism. Nature Chemical Biology 9 97-104. (doi:10.1038/ nchembio.1136)

Haslam DW \& James WP 2005 Obesity. Lancet 366 1197-1209. (doi:10.1016/S0140-6736(05)67483-1)

Hellerstrom C \& Swenne I 1991 Functional maturation and proliferation of fetal pancreatic $\beta$-cells. Diabetes 40(Suppl 2) 89-93. (doi:10.2337/ diab.40.2.S89)

Hove JR, Koster RW, Forouhar AS, Acevedo-Bolton G, Fraser SE \& Gharib M 2003 Intracardiac fluid forces are an essential epigenetic factor for embryonic cardiogenesis. Nature 421 172-177. (doi:10.1038/ nature01282)

Huang CJ, Tu CT, Hsiao CD, Hsieh FJ \& Tsai HJ 2003 Germ-line transmission of a myocardium-specific GFP transgene reveals critical regulatory elements in the cardiac myosin light chain 2 promoter of zebrafish. Developmental Dynamics 228 30-40. (doi:10.1002/dvdy. 10356)

Jorgensen SB, Richter EA \& Wojtaszewski JF 2006 Role of AMPK in skeletal muscle metabolic regulation and adaptation in relation to exercise. Journal of Physiology 574 17-31. (doi:10.1113/jphysiol.2006.109942)

Jurczyk A, Roy N, Bajwa R, Gut P, Lipson K, Yang C, Covassin L, Racki WJ, Rossini AA, Phillips N et al. 2011 Dynamic glucoregulation and mammalian-like responses to metabolic and developmental disruption in zebrafish. General and Comparative Endocrinology $170334-345$. (doi:10.1016/j.ygcen.2010.10.010)

Kannel WB \& McGee DL 1979 Diabetes and cardiovascular disease. The Framingham study. Journal of the American Medical Association 241 2035-2038. (doi:10.1001/jama.1979.03290450033020)

Katz EB, Stenbit AE, Hatton K, DePinho R \& Charron MJ 1995 Cardiac and adipose tissue abnormalities but not diabetes in mice deficient in GLUT4. Nature 377 151-155. (doi:10.1038/377151a0)

Lawson ND \& Weinstein BM 2002 In vivo imaging of embryonic vascular development using transgenic zebrafish. Developmental Biology 248 307-318. (doi:10.1006/dbio.2002.0711)

Lehman JJ, Barger PM, Kovacs A, Saffitz JE, Medeiros DM \& Kelly DP 2000 Peroxisome proliferator-activated receptor gamma coactivator-1 promotes cardiac mitochondrial biogenesis. Journal of Clinical Investigation 106 847-856. (doi:10.1172/JCI10268)

Liang Q, De Windt LJ, Witt SA, Kimball TR, Markham BE \& Molkentin JD 2001 The transcription factors GATA4 and GATA6 regulate cardiomyocyte hypertrophy in vitro and in vivo. Journal of Biological Chemistry 276 30245-30253. (doi:10.1074/jbc.M102174200)
Lopez AD, Mathers CD, Ezzati M, Jamison DT \& Murray CJ 2006 Global and regional burden of disease and risk factors, 2001: systematic analysis of population health data. Lancet 367 1747-1757. (doi:10.1016/S0140-6736(06)68770-9)

Lorell BH \& Grossman W 1987 Cardiac hypertrophy: the consequences for diastole. Journal of the American College of Cardiology 9 1189-1193. (doi:10.1016/S0735-1097(87)80326-1)

Louis A, Muffato M \& Roest Crollius H 2013 Genomicus: five genome browsers for comparative genomics in eukaryota. Nucleic Acids Research 41 D700-D705. (doi:10.1093/nar/gks1156)

Macheda ML, Kelly DJ, Best JD \& Rogers S 2002 Expression during rat fetal development of GLUT12 - a member of the class III hexose transporter family. Anatomy and Embryology 205 441-452. (doi:10.1007/s00429-002-0263-8)

Marín-Juez R, Capilla E, Carvalho-Simoes F, Camps M \& Planas JV 2014a Structural and functional evolution of glucose transporter 4 (GLUT4): a look at GLUT4 in fish. In Glucose Homeostasis. Ed L Szablewski. InTech: Rijeka, Croatia. (doi:10.5772/58094)

Marín-Juez R, Jong-Raadsen S, Yang S \& Spaink HP 2014b Hyperinsulinemia induces insulin resistance and immune suppression via Ptpn6/Shp1 in zebrafish. Journal of Endocrinology 222 229-241. (doi:10.1530/JOE-14-0178)

Mootha VK, Lindgren CM, Eriksson KF, Subramanian A, Sihag S, Lehar J, Puigserver P, Carlsson E, Ridderstrale M, Laurila E et al. 2003 PGC$1 \alpha$-responsive genes involved in oxidative phosphorylation are coordinately downregulated in human diabetes. Nature Genetics 34 267-273. (doi:10.1038/ng1180)

Pagidipati NJ \& Gaziano TA 2013 Estimating deaths from cardiovascular disease: a review of global methodologies of mortality measurement. Circulation 127 749-756. (doi:10.1161/CIRCULATIONAHA.112.128413)

Patti ME, Butte AJ, Crunkhorn S, Cusi K, Berria R, Kashyap S, Miyazaki Y, Kohane I, Costello M, Saccone R et al. 2003 Coordinated reduction of genes of oxidative metabolism in humans with insulin resistance and diabetes: potential role of PGC1 and NRF1. PNAS 100 8466-8471. (doi:10.1073/pnas.1032913100)

Puigserver P \& Spiegelman BM 2003 Peroxisome proliferator-activated receptor- $\gamma$ coactivator $1 \alpha(\mathrm{PGC}-1 \alpha)$ : transcriptional coactivator and metabolic regulator. Endocrine Reviews 24 78-90. (doi:10.1210/er. 2002-0012)

Pujol-Gimenez J, Martisova E, Perez-Mediavilla A, Lostao MP \& Ramirez MJ 2014 Expression of the glucose transporter GLUT12 in Alzheimer's disease patients. Journal of Alzheimer's Disease 42 97-101. (doi:10.3233/ JAD-132498)

Purcell SH, Aerni-Flessner LB, Willcockson AR, Diggs-Andrews KA, Fisher SJ \& Moley KH 2011 Improved insulin sensitivity by GLUT12 overexpression in mice. Diabetes 60 1478-1482. (doi:10.2337/db11-0033)

Razeghi P, Young ME, Alcorn JL, Moravec CS, Frazier OH \& Taegtmeyer H 2001 Metabolic gene expression in fetal and failing human heart. Circulation 104 2923-2931. (doi:10.1161/hc4901.100526)

Rogers S, Macheda ML, Docherty SE, Carty MD, Henderson MA, Soeller WC, Gibbs EM, James DE \& Best JD 2002 Identification of a novel glucose transporter-like protein-GLUT-12. American Journal of Physiology. Endocrinology and Metabolism 282 E733-E738.

Santalucia T, Camps M, Castello A, Munoz P, Nuel A, Testar X, Palacin M \& Zorzano A 1992 Developmental regulation of GLUT-1 (erythroid/Hep G2) and GLUT-4 (muscle/fat) glucose transporter expression in rat heart, skeletal muscle, and brown adipose tissue. Endocrinology 130 837-846. (doi:10.1210/endo.130.2.1370797)

Scheepers A, Joost HG \& Schurmann A 2004 The glucose transporter families SGLT and GLUT: molecular basis of normal and aberrant function. JPEN. Journal of Parenteral and Enteral Nutrition 28 364-371. (doi:10.1177/0148607104028005364)

Schertzer JD, Tamrakar AK, Magalhaes JG, Pereira S, Bilan PJ, Fullerton MD, Liu Z, Steinberg GR, Giacca A, Philpott DJ et al. 2011 NOD1 activators link innate immunity to insulin resistance. Diabetes $602206-2215$. (doi:10.2337/db11-0004) 
Seth A, Stemple DL \& Barroso I 2013 The emerging use of zebrafish to model metabolic disease. Disease Models \& Mechanisms 6 1080-1088. (doi:10.1242/dmm.011346)

Sliwa K, Damasceno A \& Mayosi BM 2005 Epidemiology and etiology of cardiomyopathy in Africa. Circulation 112 3577-3583. (doi:10.1161/ CIRCULATIONAHA.105.542894)

Stenbit AE, Burcelin R, Katz EB, Tsao TS, Gautier N, Charron MJ \& Le Marchand-Brustel Y 1996 Diverse effects of Glut 4 ablation on glucose uptake and glycogen synthesis in red and white skeletal muscle. Journal of Clinical Investigation 98 629-634. (doi:10.1172/JCI118833)

Stenbit AE, Tsao TS, Li J, Burcelin R, Geenen DL, Factor SM, Houseknecht K, Katz EB \& Charron MJ 1997 GLUT4 heterozygous knockout mice develop muscle insulin resistance and diabetes. Nature Medicine $\mathbf{3}$ 1096-1101. (doi:10.1038/nm1097-1096)

Stuart CA, Howell ME, Zhang Y \& Yin D 2009 Insulin-stimulated translocation of glucose transporter (GLUT) 12 parallels that of GLUT4 in normal muscle. Journal of Clinical Endocrinology and Metabolism 94 3535-3542. (doi:10.1210/jc.2009-0162)

Swoap SJ, Weinshenker D, Palmiter RD \& Garber G 2004 Dbh(-/-) mice are hypotensive, have altered circadian rhythms, and have abnormal responses to dieting and stress. American Journal of Physiology. Regulatory, Integrative and Comparative Physiology 286 R108-R113. (doi:10.1152/ajpregu.00405.2003)

Tachibana K, Jones N, Dumont DJ, Puri MC \& Bernstein A 2005 Selective role of a distinct tyrosine residue on Tie2 in heart development and early hematopoiesis. Molecular and Cellular Biology 25 4693-4702. (doi:10.1128/MCB.25.11.4693-4702.2005)

Tessadori F, van Weerd JH, Burkhard SB, Verkerk AO, de Pater E, Boukens BJ, Vink A, Christoffels VM \& Bakkers J 2012 Identification and functional characterization of cardiac pacemaker cells in zebrafish. PLOS ONE $\mathbf{7}$ e47644. (doi:10.1371/journal.pone.0047644)

Thisse B \& Thisse C 2004 Fast release clones: a high throughput expression analysis. ZFIN Direct Data Submission ZFIN ID: ZDB-PUB-040907-1. (available at: http://zfin.org/cgi-bin/webdriver?MIval=aa-pubview2. apg\&OID=ZDB-PUB-040907-1)

Tseng YC, Chen RD, Lee JR, Liu ST, Lee SJ \& Hwang PP 2009 Specific expression and regulation of glucose transporters in zebrafish ionocytes. American Journal of Physiology. Regulatory, Integrative and Comparative Physiology 297 R275-R290. (doi:10.1152/ajpregu. 00180.2009)

Vermot J, Forouhar AS, Liebling M, Wu D, Plummer D, Gharib M \& Fraser SE 2009 Reversing blood flows act through klf2a to ensure normal valvulogenesis in the developing heart. PLoS Biology 7 e1000246. (doi:10.1371/journal.pbio.1000246)

Viollet B, Guigas B, Sanz Garcia N, Leclerc J, Foretz M \& Andreelli F 2012 Cellular and molecular mechanisms of metformin: an overview. Clinical Science 122 253-270. (doi:10.1042/CS20110386)

van der Vusse GJ, van Bilsen M \& Glatz JF 2000 Cardiac fatty acid uptake and transport in health and disease. Cardiovascular Research $\mathbf{4 5}$ 279-293. (doi:10.1016/S0008-6363(99)00263-1)

Waller AP, George M, Kalyanasundaram A, Kang C, Periasamy M, Hu K \& Lacombe VA 2013 GLUT12 functions as a basal and insulinindependent glucose transporter in the heart. Biochimica et Biophysica Acta 1832 121-127. (doi:10.1016/j.bbadis.2012.09.013)

Ware B, Bevier M, Nishijima Y, Rogers S, Carnes CA \& Lacombe VA 2011 Chronic heart failure selectively induces regional heterogeneity of insulin-responsive glucose transporters. American Journal of Physiology. Regulatory, Integrative and Comparative Physiology 301 R1300-R1306. (doi:10.1152/ajpregu.00822.2010)

Zhang X, Azhar G, Chai J, Sheridan P, Nagano K, Brown T, Yang J, Khrapko K, Borras AM, Lawitts J et al. 2001 Cardiomyopathy in transgenic mice with cardiac-specific overexpression of serum response factor. American Journal of Physiology. Heart and Circulatory Physiology 280 H1782-H1792.

Zhou G, Myers R, Li Y, Chen Y, Shen X, Fenyk-Melody J, Wu M, Ventre J, Doebber T, Fujii N et al. 2001 Role of AMP-activated protein kinase in mechanism of metformin action. Journal of Clinical Investigation 108 1167-1174. (doi:10.1172/JCI13505)

Zuliani G, Galvani M, Maggio M, Volpato S, Bandinelli S, Corsi AM, Lauretani F, Cherubini A, Guralnik JM, Fellin R et al. 2010 Plasma soluble gp130 levels are increased in older subjects with metabolic syndrome. The role of insulin resistance. Atherosclerosis 213 319-324. (doi:10.1016/j.atherosclerosis.2010.08.074)

Received in final form 9 October 2014

Accepted 17 October 2014

Accepted Preprint published online 17 October 2014
() 2015 Society for Endocrinology Printed in Great Britain 